\title{
Studies on Heterosis and Heterobeltiosis in Selected Quantitative Traits during Summer Season in Bivoltine Silkworm, Bombyx mori
}

\author{
Mir Nisar Ahmad ${ }^{1 *}$, Bharath Kumar Neelaboina ${ }^{2}$, Shivkumar ${ }^{2}$, Kiran $\mathbf{R}^{3}$, Sukhen Roy Chowdhury ${ }^{4}$ \\ ${ }^{1}$ Scientist D, Silkworm Division, Central Sericultural Research and Training Institute, Central Silk Board, J\&K, India \\ ${ }^{2}$ Scientist C, Silkworm Division, Central Sericultural Research and Training Institute, Central Silk Board, J\&K, India \\ ${ }^{3}$ Scientist B, Silkworm Division, Central Sericultural Research and Training Institute, Central Silk Board, J\&K, India \\ ${ }^{4}$ Director, Central Sericultural Research and Training Institute (CSR\&TI), Central Silk Board, Jammu \& Kashmir, India
}

*Address for Correspondence: Dr. Mir Nisar Ahmad, Scientist-D, Silkworm Division, Central Sericultural Research and Training Institute (CSR\&TI), Central Silk Board, Gallandar, Pampore-192121, Jammu \& Kashmir, India

E-mail: mirnisarahmad@gmail.com

Received: 17 May 2020/ Revised: 19 Aug 2020/ Accepted: 20 Oct 2020

\begin{abstract}
Background: The phenomenon of heterosis has been commercially exploited in agriculture, horticulture, animal husbandry, piggery, poultry and sericulture. Heterosis is the phenotypic result of gene interaction in heterozygotes and the degree of heterosis increases as the genetic similarities between two parent's decreases.

Methods: The present study was carried out at Central Sericultural Research and Training Institute (CSR\&TI), Central Silk Board, Gallandar, Pampore during summer 2019 (July-August) and extent of heterosis was studied in six quantitative traits viz., Cocoon yield per 10000 larvae by weight, Single cocoon weight, Single shell weight, Shell ratio, Filament length and Pupation rate in a set of 30 bivoltine silkworm hybrids along with three control hybrids. The data generated were analysed statistically and subjected to multiple trait Evaluation index (E.I).

Results: The average relative heterosis values ranged from-5.59 (DUN6 X DUN22) to 4.94 (S8 X CSR27). Among the 30 hybrids studied only 13 hybrids showed a variable amount of relative heterosis. The average heterobeltiosis values ranged from -6.90 (Pam117 X DUN 22) to 3.08 (S8 X CSR27). Among the 30 hybrids, only 8 hybrids showed a variable amount of heterobeltiosis. Maximum relative heterosis and heterobeltiosis have been observed in filament length $(17.14 \& 14.73$, respectively).

Conclusion: Hybrids having CSR 27, Pam 117, S8 and N5 as one of the parents have shown higher heterosis and heterobeltiosis. Among the hybrids S 8 X CSR27 and CSR27 X Pam117 have shown higher relative heterosis and heterobeltiosis. The results have also been confirmed through evaluation index values calculated on relative heterosis and heterobeltiosis.
\end{abstract}

Key-words: Bombyx mori, Bivoltine silkworm, Evaluation index, Heterobeltiosis, Relative heterosis

\section{INTRODUCTION}

The silkworm, B. mori L (Lepidoptera: Bombycidae) is an insect of great importance for its production of silk, aptly named the queen of natural fibres ${ }^{[1]}$. This unique lepidopteran insect completes its life cycle while engineering an economically distinct structure made of silk called cocoon ${ }^{[2]}$.

\footnotetext{
How to cite this article

Ahmad MN, Neelaboina BK, kumar S, Kiran R, Chowdhury SR. Studies on Heterosis and Heterobeltiosis in Selected Quantitative Traits during Summer Season in Bivoltine Silkworm, Bombyx mori L.. SSR Inst. Int. J. Life Sci., 2020; 6(6): 2694-2703.
}

Access this article online https://iijls.com/
The lepidopteran species have been reared for silk production for more than 5000 years ${ }^{[3]}$. Since then, silkworms have undergone many evolutionary changes due to natural as well as manmade selections after several thousands of generations, thereby creating a wide genetic diversity ${ }^{[4]}$.

Since so many years sericulture has proved to have considerable socio-economic relevance in India due to its suitability to small and marginal farmers and also due to low-cost investment.

The major commercial silkworm rearing is being practised under temperate climatic conditions of Kashmir division of the Union Territory of Jammu \& Kashmir during the spring season (May-June) only and 
about $15-20 \%$ rearers conduct second rearing during the autumn season (August-September). It has been observed that many rearers due to one reason or the other are unable to conduct rearing during the spring season and are interested in conducting rearing during summer season ${ }^{[5]}$ (July-August) on the left overleaf of the spring season. It is to mention here that from a single set of the mulberry plantation, under recommended pruning schedule for Kashmir climatic conditions, the leaf is available for two rearings in a year i.e., spring and autumn. As summer season can also be exploited for a limited commercial rearing on left overleaf of spring flush or from a separate set of mulberry plantation pruned for harvesting leaf during the summer season ${ }^{[6]}$, need was felt to identify silkworm hybrids suitable for rearing during the summer season. The Kashmir weather averages during summer months are depicted in Table 1.

Table 1: Kashmir Weather Averages for June, July and August 2019

\begin{tabular}{ccccccc}
\hline Months & $\begin{array}{c}\text { Min. } \\
\text { Temp. }\left({ }^{\circ} \mathrm{C}\right)\end{array}$ & $\begin{array}{c}\text { Max. } \\
\text { Temp. } \\
\left({ }^{\circ} \mathrm{C}\right)\end{array}$ & $\begin{array}{c}\text { Average } \\
\text { Temp. }\left({ }^{\circ} \mathrm{C}\right)\end{array}$ & $\begin{array}{c}\text { Average } \\
\text { Precipitation/ } \\
\text { Rainfall }(\mathrm{mm})\end{array}$ & $\begin{array}{c}\text { Avg. Sunlight } \\
\text { hours/Day }\end{array}$ & $\begin{array}{c}\text { Relative Humidity } \\
\text { (\%) }\end{array}$ \\
\hline June & 14.0 & 29.0 & 21.5 & 36 & 7.5 & 57 \\
July & 18.0 & 31.0 & 24.5 & 61 & 8.0 & 62 \\
August & 18.0 & 30.0 & 24.0 & 62 & 7.5 & 67 \\
\hline
\end{tabular}

\section{METHODS AND METHODS}

The present study was carried out at Central Sericultural Research and Training Institute (CSR\&TI), Central Silk Board, Gallandar, Pampore during summer, 2019 (JulyAugust). Six silkworm parental races viz., CSR27, Pam 117, S8, N5, DUN22 and DUN6 were selected based on their better performance during the summer season. The six races were crossed (Full diallel) resulting in 30 hybrid combinations. The extent of heterosis was studied in six quantitative traits viz., Cocoon yield per 10000 larvae by weight, Single cocoon weight, Single shell weight, Shell ratio, Filament length and Pupation rate in these 30 bivoltine silkworms (B. mori L.) hybrids along with three control hybrids viz., SH6 X NB4D2, CSR6 X CSR26 and CSR2 $X$ CSR27. The standard rearing techniques were followed ${ }^{[7]}$. The parental races were reared in three replications each and the hybrids were reared in two replications per hybrid. 250 larvae were retained in each replication after $3^{\text {rd }}$ moult.

The data generated for each trait was pooled and analysed to evaluate the performance of the parents as well as the degree of manifestation of heterosis and heterobeltiosis in the hybrids. The heterosis and heterobeltiosis in the $\mathrm{F} 1$ crosses were calculated by using the following formula:

$$
\text { Heterosis }=\underline{\mathrm{F} 1-\mathrm{MPV}} \times 10
$$

\section{Heterobeltiosis $=\underline{\mathrm{F} 1-\mathrm{BPV}} \times 100$ BPV}

Where,

$\mathrm{F} 1=$ Mean of the hybrid; MPV= Mid parent value

$\mathrm{BPV}=$ Better parent value

Evaluation index values were calculated on the values of heterosis and heterobeltiosis obtained for all the traits. The evaluation index (EI) was ca1cuated as per the below-mentioned procedure ${ }^{[8]}$.

$$
\text { Evaluation Index }=\frac{A-B}{C} \times 10+50
$$

Where,

$A=$ Value obtained for a trait in a breed; $B=$ Mean value of a trait of all the breed; $C=$ Standard deviation of a trait of all the breeds; $10=$ Standard unit; $50=$ Fixed value

The index value obtained for all the traits was combined and the average El values were obtained. The El value fixed for the selection of a line is 50 or $>50$. The genotype, which scored above the limit, is considered to possess greater economic value.

\section{RESULTS}

The average rearing and reeling performance of the parental races and the $\mathrm{F} 1$ crosses are presented in Table 2 and 3. 
Table 2: Average rearing and reeling performance of the parental races during summer, 2019

\begin{tabular}{cccccccc}
\hline S.No. & Race & $\begin{array}{c}\text { Cocoon Yield/10000 } \\
\text { larvae by wt. (kg) }\end{array}$ & $\begin{array}{c}\text { Single } \\
\text { Cocoon } \\
\text { Weight(g) }\end{array}$ & $\begin{array}{c}\text { Single Shell } \\
\text { Weight(g) }\end{array}$ & $\begin{array}{c}\text { Shell } \\
\text { Ratio } \\
\text { (\%) }\end{array}$ & $\begin{array}{c}\text { Filament } \\
\text { Length } \\
\text { (m) }\end{array}$ & $\begin{array}{c}\text { Pupation } \\
\text { rate } \\
\text { (\%) }\end{array}$ \\
\hline 1 & CSR 27 & 13.17 & 1.63 & 0.33 & 20.21 & 768 & 88.00 \\
2 & Pam 117 & 13.87 & 1.67 & 0.34 & 20.14 & 855 & 92.00 \\
3 & S 8 & 13.24 & 1.55 & 0.32 & 20.32 & 860 & 89.00 \\
4 & N 5 & 13.80 & 1.64 & 0.33 & 20.07 & 859 & 85.00 \\
5 & DUN 22 & 14.90 & 1.62 & 0.32 & 19.81 & 801 & 86.00 \\
6 & DUN 6 & 14.18 & 1.69 & 0.33 & 19.22 & 776 & 89.00 \\
7 & CSR 2 & 13.70 & 1.65 & 0.34 & 20.30 & 835 & 85.00 \\
8 & CSR 6 & 12.52 & 1.63 & 0.32 & 19.81 & 784 & 84.00 \\
9 & CSR 26 & 13.84 & 1.63 & 0.33 & 19.99 & 862 & 87.00 \\
10 & SH 6 & 14.20 & 1.70 & 0.31 & 17.96 & 744 & 92.00 \\
11 & NB4D2 & 13.80 & 1.56 & 0.29 & 18.26 & 776 & 92.00 \\
& MEAN & 13.75 & 1.63 & 0.32 & 19.64 & 810.91 & 88.09 \\
& SD & 0.62 & 0.05 & 0.01 & 0.82 & 44.09 & 2.98 \\
\hline
\end{tabular}

Table 3: Average rearing and reeling performance of hybrids during summer, 2019

\begin{tabular}{clcccccc}
\hline S.No. & \multicolumn{1}{c}{ Hybrid } & $\begin{array}{c}\text { Cocoon } \\
\text { Yield/10000 } \\
\text { larvae by } \\
\text { wt.(kg) }\end{array}$ & $\begin{array}{c}\text { Single } \\
\text { Cocoon } \\
\text { Weight } \\
\text { (g) }\end{array}$ & $\begin{array}{c}\text { Single } \\
\text { Shell } \\
\text { Weight } \\
\text { (g) }\end{array}$ & $\begin{array}{c}\text { Shell } \\
\text { Ratio } \\
\text { (\%) }\end{array}$ & $\begin{array}{c}\text { Filament } \\
\text { Length } \\
\text { (m) }\end{array}$ & $\begin{array}{c}\text { Pupation } \\
\text { rate } \\
\text { (\%) }\end{array}$ \\
\hline 1 & & 15.38 & 1.63 & 0.34 & 20.57 & 869 & 92.00 \\
2 & CSR 27 X Pam 117 & 13.84 & 1.57 & 0.30 & 19.06 & 813 & 85.00 \\
3 & CSR 27 X S 8 & 14.74 & 1.60 & 0.29 & 18.17 & 844 & 92.00 \\
4 & Pam 117 X CSR 27 & 14.44 & 1.60 & 0.34 & 20.97 & 859 & 95.00 \\
5 & Pam 117 X S 8 & 13.84 & 1.65 & 0.32 & 19.43 & 809 & 86.00 \\
6 & Pam 117 X N 5 & 14.50 & 1.67 & 0.35 & 20.92 & 904 & 91.00 \\
7 & S 8 X CSR 27 & 14.18 & 1.59 & 0.33 & 20.71 & 914 & 94.00 \\
8 & S 8 X Pam 117 & 14.60 & 1.69 & 0.35 & 20.66 & 839 & 90.00 \\
9 & S 8 X N 5 & 13.78 & 1.56 & 0.30 & 18.87 & 842 & 91.00 \\
10 & N 5 X CSR 27 & 13.08 & 1.55 & 0.29 & 18.41 & 851 & 92.00 \\
11 & N 5 X Pam 117 & 13.30 & 1.50 & 0.32 & 20.96 & 927 & 93.00 \\
12 & N 5 X S 8 & 13.90 & 1.62 & 0.32 & 19.50 & 847 & 94.00 \\
13 & Pam 117 X DUN 22 & 14.50 & 1.58 & 0.29 & 18.01 & 805 & 90.00 \\
14 & Pam 117 X DUN 6 & 14.64 & 1.55 & 0.32 & 20.27 & 896 & 93.00 \\
15 & CSR 27 X DUN 22 & 14.26 & 1.51 & 0.31 & 20.50 & 919 & 93.00 \\
16 & CSR 27 X DUN 6 & 14.06 & 1.58 & 0.30 & 18.63 & 817 & 85.00 \\
17 & DUN 22 X Pam 117 & 14.42 & 1.55 & 0.29 & 18.38 & 820 & 92.00 \\
18 & DUN 22 X CSR 27 & 14.40 & 1.69 & 0.34 & 19.83 & 812 & 88.00 \\
19 & DUN 22 X DUN 6 & 13.44 & 1.63 & 0.32 & 19.31 & 821 & 85.00 \\
20 & DUN 22 X S 8 & 14.28 & 1.67 & 0.33 & 19.51 & 840 & 90.00 \\
21 & DUN 22 X N 5 & 13.60 & 1.63 & 0.32 & 19.34 & 827 & 88.00 \\
22 & DUN 6 X Pam 117 & 14.28 & 1.68 & 0.33 & 19.39 & 790 & 85.00 \\
23 & DUN 6 X CSR 27 & 13.84 & 1.63 & 0.31 & 18.99 & 774 & 87.00 \\
24 & DUN 6 X DUN 22 & 12.56 & 1.56 & 0.30 & 18.94 & 756 & 88.00 \\
\hline
\end{tabular}


crossef DOI: 10.21276/SSR-IIJLS.2020.6.6.4

\begin{tabular}{clcccccc}
\hline 25 & DUN 6 X S 8 & 13.30 & 1.49 & 0.30 & 19.73 & 903 & 94.00 \\
26 & DUN 6 X N 5 & 14.66 & 1.62 & 0.32 & 19.54 & 803 & 91.00 \\
27 & S 8 X DUN 22 & 14.68 & 1.56 & 0.29 & 18.64 & 803 & 93.00 \\
28 & S 8 X DUN 6 & 14.70 & 1.55 & 0.32 & 20.27 & 801 & 94.00 \\
29 & N 5 X DUN 22 & 14.90 & 1.62 & 0.33 & 20.07 & 819 & 90.00 \\
30 & N 5 X DUN 6 & 14.20 & 1.54 & 0.30 & 19.10 & 798 & 91.00 \\
Control 1 & SH6XNB4D2 & 14.90 & 1.55 & 0.29 & 18.73 & 818 & 95.00 \\
Control 2 & CSR6XCSR26 & 12.27 & 1.55 & 0.31 & 19.74 & 876 & 88.00 \\
Control 3 & CSR2XCSR27 & 12.80 & 1.58 & 0.32 & 20.00 & 878 & 89.00 \\
& MEAN & 14.07 & 1.59 & 0.31 & 19.55 & 839.21 & 90.42 \\
& SD & 0.71 & 0.05 & 0.02 & 0.85 & 43.52 & 3.09 \\
\hline
\end{tabular}

Heterosis- Perusal of data reveals that the extent of heterosis in cocoon yield ranged from -13.62 (DUN6 $X$ DUN22) to 13.76 (CSR27 $X$ Pam 117). The heterosis ranged from -9.37 (N5 X Pam 117) to 5.36 (DUN22 X S8) in single cocoon weight; -12.12 (N5 X CSR27 \& DUN22 X Pam 117) to 6.06 (S8 X Pam 117) in single shell weight; 9.84 (Pam 117 X DUN22) to 4.25 (N5 X Pam 117) in shell ratio; -5.66 (Pam $117 \times$ S8) to 17.14 (CSR27 X DUN22) in filament length and -6.08 (DUN6 X Pam 117) to 8.05 (N5 $X$ S8) in pupation rate. The average relative heterosis values ranged from-5.59 (DUN6 X DUN22) to 4.94 (S8 X CSR27) (Table 4). Evaluation index was also calculated on the values of relative heterosis (Table 5). Data reveals that the same hybrids ranked in the first six positions based on the values of average relative heterosis as well as the evaluation index values.

Table 4: Heterosis values of the hybrids during summer, 2019

\begin{tabular}{lccccccc}
\hline \multicolumn{1}{c}{ Hybrids } & $\begin{array}{c}\text { Cocoon } \\
\text { Yield by } \\
\text { wt. }\end{array}$ & $\begin{array}{c}\text { Single } \\
\text { Cocoon } \\
\text { Weight }\end{array}$ & $\begin{array}{c}\text { Single } \\
\text { Shell } \\
\text { Weight }\end{array}$ & $\begin{array}{c}\text { Shell } \\
\text { Ratio }\end{array}$ & $\begin{array}{c}\text { Filament } \\
\text { Length }\end{array}$ & $\begin{array}{c}\text { Pupation } \\
\text { rate }\end{array}$ & $\begin{array}{c}\text { Avg. } \\
\text { Heterosis }\end{array}$ \\
\hline CSR 27 X Pam 117 & 13.76 & -1.21 & 1.49 & 1.96 & 7.09 & 2.22 & 4.22 \\
CSR 27 X S 8 & 4.81 & -1.26 & -7.69 & -5.95 & -0.12 & -3.95 & -2.36 \\
CSR 27 X N 5 & 9.31 & -2.14 & -12.12 & -9.78 & 3.75 & 6.36 & -0.77 \\
Pam 117 X CSR 27 & 6.80 & -3.03 & 1.49 & 3.94 & 5.85 & 5.56 & 3.44 \\
Pam 117 X S 8 & 2.10 & 2.48 & -3.03 & -3.95 & -5.66 & -4.97 & -2.17 \\
Pam 117 X N 5 & 4.81 & 0.91 & 4.48 & 4.05 & 5.48 & 2.82 & 3.76 \\
S 8 X CSR 27 & 7.38 & 0.00 & 1.54 & 2.20 & 12.29 & 6.21 & 4.94 \\
S 8 X Pam 117 & 7.71 & 4.97 & 6.06 & 2.13 & -2.16 & -0.55 & 3.03 \\
S 8 X N 5 & 1.92 & -2.19 & -7.69 & -6.56 & -2.04 & 4.60 & -1.99 \\
N 5 X CSR 27 & -3.00 & -5.20 & -12.12 & -8.59 & 4.61 & 6.36 & -2.99 \\
N 5 X Pam 117 & -3.87 & -9.37 & -4.48 & 4.25 & 8.17 & 5.08 & -0.04 \\
N 5 X S 8 & 2.81 & 1.57 & -1.54 & -3.44 & -1.45 & 8.05 & 1.00 \\
Pam 117 X DUN 22 & 0.80 & -3.95 & -12.12 & -9.84 & -2.78 & 1.12 & -4.46 \\
Pam 117 X DUN 6 & 4.39 & -7.74 & -4.48 & 3.00 & 9.87 & 2.76 & 1.30 \\
CSR 27 X DUN 22 & 1.60 & -7.08 & -4.62 & 2.45 & 17.14 & 6.90 & 2.73 \\
CSR 27 X DUN 6 & 2.82 & -4.82 & -9.09 & -5.50 & 5.83 & -3.95 & -2.45 \\
DUN 22 X Pam 117 & 0.24 & -5.78 & -12.12 & -7.98 & -0.97 & 3.37 & -3.87 \\
DUN 22 X CSR 27 & 2.60 & 4.00 & 4.62 & -0.90 & 3.51 & 1.15 & 2.50 \\
DUN 22 X DUN 6 & -7.57 & -1.51 & -1.54 & -1.05 & 4.12 & -2.86 & -1.74 \\
DUN 22 X S 8 & 1.49 & 5.36 & 3.13 & -2.77 & 1.14 & 2.86 & 1.87 \\
DUN 22 X N 5 & -5.23 & 0.00 & -1.54 & -3.01 & -0.36 & 2.92 & -1.20 \\
DUN 6 X Pam 117 & 1.82 & 0.00 & -1.49 & -1.47 & -3.13 & -6.08 & -1.73 \\
\hline
\end{tabular}




\begin{tabular}{lccccccc}
\hline DUN 6 X CSR 27 & 1.21 & -1.81 & -6.06 & -3.68 & 0.26 & -1.69 & -1.96 \\
DUN 6 X DUN 22 & -13.62 & -5.74 & -7.69 & -2.95 & -4.12 & 0.57 & -5.59 \\
DUN 6 X S 8 & -2.99 & -8.02 & -7.69 & -0.20 & 10.39 & 5.62 & -0.48 \\
DUN 6 X N 5 & 4.79 & -2.70 & -3.03 & -0.53 & -1.77 & 4.60 & 0.23 \\
S 8 X DUN 22 & 4.34 & -1.58 & -9.38 & -7.10 & -3.31 & 6.29 & -1.79 \\
S 8 X DUN 6 & 7.22 & -4.32 & -1.54 & 2.53 & -2.08 & 5.62 & 1.24 \\
N 5 X DUN 22 & 3.83 & -0.61 & 1.54 & 0.65 & -1.33 & 5.26 & 1.56 \\
N 5 X DUN 6 & 1.50 & -7.51 & -9.09 & -2.77 & -2.39 & 4.60 & -2.61 \\
SH6XNB4D2 & 6.43 & -4.91 & -3.33 & 3.42 & 7.63 & 3.26 & 2.08 \\
CSR6XCSR26 & -6.90 & -4.91 & -4.62 & -0.80 & 6.44 & 2.92 & -1.31 \\
CSR2XCSR27 & -4.76 & -3.66 & -4.48 & -1.26 & 9.54 & 2.89 & -0.29 \\
MEAN & 1.77 & -2.48 & -3.89 & -1.80 & 2.71 & 2.60 & -0.18 \\
MAXIMUM & 13.76 & 5.36 & 6.06 & 4.25 & 17.14 & 8.05 & 4.94 \\
MINIMUM & -13.62 & -9.37 & -12.12 & -9.84 & -5.66 & -6.08 & -5.59 \\
SD & 5.49 & 3.73 & 5.18 & 4.18 & 5.55 & 3.72 & 2.64 \\
\hline
\end{tabular}

Table 5: Evaluation Index on Heterosis values of the hybrids during summer, 2019

\begin{tabular}{|c|c|c|c|c|c|c|c|}
\hline Hybrid & $\begin{array}{c}\text { Cocoon } \\
\text { Yield by } \\
\text { wt. }\end{array}$ & $\begin{array}{l}\text { Single } \\
\text { Cocoon } \\
\text { Weight }\end{array}$ & $\begin{array}{c}\text { Single } \\
\text { Shell } \\
\text { Weight }\end{array}$ & $\begin{array}{l}\text { Shell } \\
\text { Ratio }\end{array}$ & $\begin{array}{c}\text { Filament } \\
\text { Length }\end{array}$ & $\begin{array}{l}\text { Pupation } \\
\text { rate }\end{array}$ & $\begin{array}{c}\text { Avg. } \\
\text { Heterosis }\end{array}$ \\
\hline CSR 27 X Pam 117 & 71.84 & 53.40 & 60.39 & 61.39 & 57.89 & 48.98 & 66.66 \\
\hline CSR $27 \times$ S 8 & 55.54 & 53.27 & 42.66 & 42.46 & 44.90 & 32.39 & 41.74 \\
\hline CSR $27 \times N 5$ & 63.73 & 50.91 & 34.11 & 33.30 & 51.87 & 60.11 & 47.77 \\
\hline Pam 117 X CSR 27 & 59.16 & 48.53 & 60.39 & 66.12 & 55.66 & 57.96 & 63.69 \\
\hline Pam 117 X S 8 & 50.60 & 63.30 & 51.66 & 47.25 & 34.92 & 29.65 & 42.46 \\
\hline Pam 117 X N 5 & 55.54 & 59.09 & 66.16 & 66.39 & 54.99 & 50.59 & 64.92 \\
\hline S $8 \times$ CSR 27 & 60.22 & 56.65 & 60.48 & 61.96 & 67.26 & 59.70 & 69.38 \\
\hline S 8 X Pam 117 & 60.82 & 69.97 & 69.21 & 61.79 & 41.23 & 41.53 & 62.15 \\
\hline S $8 \times N 5$ & 50.27 & 50.78 & 42.66 & 41.00 & 41.44 & 55.38 & 43.13 \\
\hline N $5 \times \operatorname{CSR} 27$ & 41.31 & 42.71 & 34.11 & 36.15 & 53.42 & 60.11 & 39.36 \\
\hline N 5 X Pam 117 & 39.73 & 31.53 & 48.86 & 66.87 & 59.84 & 56.67 & 50.54 \\
\hline N $5 \times 58$ & 51.89 & 60.86 & 54.54 & 48.47 & 42.50 & 64.65 & 54.47 \\
\hline Pam 117 X DUN 22 & 48.23 & 46.06 & 34.11 & 33.16 & 40.11 & 46.02 & 33.78 \\
\hline Pam 117 X DUN 6 & 54.77 & 35.90 & 48.86 & 63.88 & 62.90 & 50.43 & 55.61 \\
\hline CSR 27 X DUN 22 & 49.69 & 37.67 & 48.59 & 62.56 & 76.00 & 61.56 & 61.03 \\
\hline CSR 27 X DUN 6 & 51.91 & 43.73 & 39.96 & 43.54 & 55.62 & 32.39 & 41.40 \\
\hline DUN 22 X Pam 117 & 47.21 & 41.15 & 34.11 & 37.61 & 43.37 & 52.07 & 36.01 \\
\hline DUN 22 X CSR 27 & 51.51 & 67.37 & 66.43 & 54.55 & 51.44 & 46.10 & 60.14 \\
\hline DUN 22 X DUN 6 & 32.99 & 52.60 & 54.54 & 54.19 & 52.54 & 35.32 & 44.11 \\
\hline DUN 22 XS 8 & 49.49 & 71.02 & 63.55 & 50.07 & 47.17 & 50.70 & 57.76 \\
\hline DUN 22 X N 5 & 37.25 & 56.65 & 54.54 & 49.50 & 44.47 & 50.86 & 46.12 \\
\hline DUN 6 X Pam 117 & 50.09 & 56.65 & 54.63 & 53.18 & 39.48 & 26.67 & 44.15 \\
\hline DUN 6 X CSR 27 & 48.98 & 51.80 & 45.81 & 47.89 & 45.59 & 38.47 & 43.25 \\
\hline DUN 6 X DUN 22 & 21.97 & 41.26 & 42.66 & 49.64 & 37.69 & 44.54 & 29.50 \\
\hline DUN 6 XS 8 & 41.33 & 35.15 & 42.66 & 56.22 & 63.84 & 58.12 & 48.86 \\
\hline DUN $6 \times N 5$ & 55.50 & 49.41 & 51.66 & 55.43 & 41.93 & 55.38 & 51.54 \\
\hline
\end{tabular}


crossef DOI: $10.21276 /$ SSR-IIJLS.2020.6.6.4

\begin{tabular}{llllllll}
\hline S 8 X DUN 22 & 54.68 & 52.41 & 39.40 & 39.71 & 39.15 & 59.92 & 43.90 \\
S 8 X DUN 6 & 59.93 & 45.07 & 54.54 & 62.75 & 41.37 & 58.12 & 55.37 \\
N 5 X DUN 22 & 53.75 & 55.01 & 60.48 & 58.25 & 42.72 & 57.15 & 56.58 \\
N 5 X DUN 6 & 49.51 & 36.51 & 39.96 & 50.07 & 40.81 & 55.38 & 40.80 \\
SH6XNB4D2 & 58.49 & 43.49 & 51.08 & 64.88 & 58.86 & 51.77 & 58.57 \\
CSR6XCSR26 & 34.21 & 43.49 & 48.59 & 54.78 & 56.72 & 50.86 & 45.71 \\
CSR2XCSR27 & 38.11 & 46.84 & 48.86 & 53.68 & 62.31 & 50.78 & 49.59 \\
\hline
\end{tabular}

Heterobeltiosis- Perusal of data reveals that the extent of heterobeltiosis in cocoon yield ranged from -15.70 (DUN6 X DUN22) to 10.89 (CSR27 X Pam 117). The heterobeltiosis ranged from -11.83 (DUN6 X S8) to 3.68 (DUN22 $X$ CSR27) in single cocoon weight; -14.71 (DUN22 $X$ Pam 117 \& Pam 117 X DUN22) to 3.13 (DUN22 X S8) in single shell weight; -10.58 (Pam $117 \times$ DUN22) to 4.07 (N5 X Pam 117) in shell ratio; -7.60 (DUN6 X Pam 117) to 14.73 (CSR27 $X$ DUN22) in filament length and -7.61
(DUN6 X Pam 117) to 5.68 (CSR27 X DUN22) in pupation rate. The average heterobeltiosis values ranged from6.90 (Pam 117 X DUN22) to 3.08 (S8 X CSR27) (Table 6). Evaluation index was also calculated on the values of heterobeltiosis (Table 7). Data reveals that the same hybrids ranked in the first six positions based on the values of average heterobeltiosis as well as the evaluation index values.

Table 6: Heterobeltiosis values of the hybrids during summer, 2019

\begin{tabular}{lccccccc}
\hline \multicolumn{1}{c}{ Hybrids } & $\begin{array}{c}\text { Cocoon } \\
\text { Yield by } \\
\text { wt. }\end{array}$ & $\begin{array}{c}\text { Single } \\
\text { Cocoon } \\
\text { Weight }\end{array}$ & $\begin{array}{c}\text { Single } \\
\text { Shell } \\
\text { Weight }\end{array}$ & $\begin{array}{c}\text { Shell } \\
\text { Ratio }\end{array}$ & $\begin{array}{c}\text { Filament } \\
\text { Length }\end{array}$ & $\begin{array}{c}\text { Pupation } \\
\text { rate }\end{array}$ & $\begin{array}{c}\text { Avg. Heterob- } \\
\text { eltiosis }\end{array}$ \\
\hline CSR 27 X Pam 117 & 10.89 & -2.40 & 0.00 & 1.78 & 1.64 & 0.00 & 1.99 \\
CSR 27 X S 8 & 4.53 & -3.68 & -9.09 & -6.20 & -5.47 & -4.49 & -4.07 \\
CSR 27 X N 5 & 6.81 & -2.44 & -12.12 & -10.09 & -1.75 & 4.55 & -2.51 \\
Pam 117 X CSR 27 & 4.11 & -4.19 & 0.00 & 3.76 & 0.47 & 3.26 & 1.24 \\
Pam 117 X S 8 & -0.22 & -1.20 & -5.88 & -4.38 & -5.93 & -6.52 & -4.02 \\
Pam 117 X N 5 & 4.54 & 0.00 & 2.94 & 3.87 & 5.24 & -1.09 & 2.58 \\
S 8 X CSR 27 & 7.10 & -2.45 & 0.00 & 1.92 & 6.28 & 5.62 & 3.08 \\
S 8 X Pam 117 & 5.26 & 1.20 & 2.94 & 1.67 & -2.44 & -2.17 & 1.08 \\
S 8 X N 5 & -0.14 & -4.88 & -9.09 & -7.14 & -2.09 & 2.25 & -3.52 \\
N 5 X CSR 27 & -5.22 & -5.49 & -12.12 & -8.91 & -0.93 & 4.55 & -4.69 \\
N 5 X Pam 117 & -4.11 & -10.18 & -5.88 & 4.07 & 7.92 & 1.09 & -1.18 \\
N 5 X S 8 & 0.72 & -1.22 & -3.03 & -4.04 & -1.51 & 5.62 & -0.58 \\
Pam 117 X DUN 22 & -2.68 & -5.39 & -14.71 & -10.58 & -5.85 & -2.17 & -6.90 \\
Pam 117 X DUN 6 & 3.24 & -8.28 & -5.88 & 0.65 & 4.80 & 1.09 & -0.73 \\
CSR 27 X DUN 22 & -4.30 & -7.36 & -6.06 & 1.43 & 14.73 & 5.68 & 0.69 \\
CSR 27 X DUN 6 & -0.85 & -6.51 & -9.09 & -7.82 & 5.28 & -4.49 & -3.91 \\
DUN 22 X Pam 117 & -3.22 & -7.19 & -14.71 & -8.74 & -4.09 & 0.00 & -6.33 \\
DUN 22 X CSR 27 & -3.36 & 3.68 & 3.03 & -1.88 & 1.37 & 0.00 & 0.47 \\
DUN 22 X DUN 6 & -9.80 & -3.55 & -3.03 & -2.52 & 2.50 & -4.49 & -3.48 \\
DUN 22 X S 8 & -4.16 & 3.09 & 3.13 & -3.99 & -2.33 & 1.12 & -0.52 \\
DUN 22 X N 5 & -8.72 & -0.61 & -3.03 & -3.64 & -3.73 & 2.33 & -2.90 \\
DUN 6 X Pam 117 & 0.71 & -0.59 & -2.94 & -3.72 & -7.60 & -7.61 & -3.63 \\
DUN 6 X CSR 27 & -2.40 & -3.55 & -8.82 & -1.20 & -0.26 & -2.25 & -3.08 \\
DUN 6 X DUN 22 & -15.70 & -7.69 & -9.09 & -1.46 & -5.62 & -1.12 & -6.78 \\
DUN 6 X S 8 & -6.21 & -11.83 & -9.09 & -2.90 & 5.00 & 5.62 & -3.24 \\
\hline
\end{tabular}


Crossef DOI: 10.21276/SSR-IIJLS.2020.6.6.4

\begin{tabular}{lccccccc}
\hline DUN 6 X N 5 & 3.39 & -4.14 & -3.03 & -2.64 & -6.52 & 2.25 & -1.78 \\
S 8 X DUN 22 & -1.48 & -3.70 & -9.38 & -8.27 & -6.63 & 4.49 & -4.16 \\
S 8 X DUN 6 & 3.67 & -8.28 & -3.03 & -0.25 & -6.86 & 5.62 & -1.52 \\
N 5 X DUN 22 & 0.00 & -1.22 & 0.00 & 0.00 & -4.66 & 4.65 & -0.21 \\
N 5 X DUN 6 & 0.14 & -8.88 & -9.09 & -4.83 & -7.10 & 2.25 & -4.59 \\
SH6XNB4D2 & 4.93 & -8.82 & -6.45 & 2.57 & 5.41 & 3.26 & 0.15 \\
CSR6XCSR26 & -11.34 & -4.91 & -6.06 & -0.35 & 1.62 & 1.15 & -3.32 \\
CSR2XCSR27 & -6.60 & -4.24 & -5.88 & -1.04 & 5.15 & 1.14 & -1.91 \\
MEAN & -0.92 & -4.15 & -5.29 & -2.57 & -0.42 & 0.95 & -2.07 \\
MAXIMUM & 10.89 & 3.68 & 3.13 & 4.07 & 14.73 & 5.68 & 3.08 \\
MINIMUM & -15.70 & -11.83 & -14.71 & -10.58 & -7.60 & -7.61 & -6.90 \\
SD & 5.81 & 3.69 & 5.03 & 4.21 & 5.40 & 3.70 & 2.62 \\
\hline
\end{tabular}

Table7: Evaluation Index on Heterobeltiosis values of the hybrids during summer, 2019

\begin{tabular}{|c|c|c|c|c|c|c|c|}
\hline Hybrids & $\begin{array}{c}\text { Cocoon } \\
\text { Yield by } \\
\text { wt. }\end{array}$ & $\begin{array}{l}\text { Single } \\
\text { Cocoon } \\
\text { Weight }\end{array}$ & $\begin{array}{c}\text { Single } \\
\text { Shell } \\
\text { Weight }\end{array}$ & $\begin{array}{l}\text { Shell } \\
\text { Ratio }\end{array}$ & $\begin{array}{l}\text { Filament } \\
\text { Length }\end{array}$ & $\begin{array}{l}\text { Pupation } \\
\text { rate }\end{array}$ & $\begin{array}{c}\text { Avg. } \\
\text { Heterob- } \\
\text { eltiosis }\end{array}$ \\
\hline CSR 27 X Pam 117 & 70.33 & 54.74 & 60.52 & 60.33 & 53.81 & 47.43 & 65.48 \\
\hline CSR $27 \times$ S 8 & 59.38 & 51.27 & 42.45 & 41.38 & 40.65 & 35.30 & 42.38 \\
\hline CSR $27 \times N 5$ & 63.30 & 54.63 & 36.42 & 32.14 & 47.54 & 59.73 & 48.33 \\
\hline Pam 117 X CSR 27 & 58.66 & 49.89 & 60.52 & 65.04 & 51.65 & 56.24 & 62.61 \\
\hline Pam 117 X S 8 & 51.20 & 57.99 & 48.83 & 45.70 & 39.80 & 29.81 & 42.55 \\
\hline Pam 117 X N 5 & 59.40 & 61.25 & 66.36 & 65.30 & 60.48 & 44.49 & 67.76 \\
\hline S $8 \times$ CSR 27 & 63.80 & 54.61 & 60.52 & 60.67 & 62.41 & 62.62 & 69.65 \\
\hline S 8 X Pam 117 & 60.64 & 64.50 & 66.36 & 60.07 & 46.26 & 41.57 & 62.01 \\
\hline S $8 \times N 5$ & 51.34 & 48.02 & 42.45 & 39.14 & 46.91 & 53.51 & 44.48 \\
\hline N $5 \times$ CSR 27 & 42.60 & 46.37 & 36.42 & 34.94 & 49.06 & 59.73 & 40.01 \\
\hline N 5 X Pam 117 & 44.51 & 33.66 & 48.83 & 65.77 & 65.44 & 50.38 & 53.39 \\
\hline N $5 \times S 8$ & 52.82 & 57.94 & 54.49 & 46.51 & 47.98 & 62.62 & 55.70 \\
\hline Pam 117 X DUN 22 & 46.97 & 46.64 & 31.27 & 30.97 & 39.94 & 41.57 & 31.58 \\
\hline Pam 117 X DUN 6 & 57.16 & 38.81 & 48.83 & 57.65 & 59.67 & 50.38 & 55.11 \\
\hline CSR 27 X DUN 22 & 44.18 & 41.30 & 48.47 & 59.50 & 78.06 & 62.78 & 60.52 \\
\hline CSR 27 X DUN 6 & 50.12 & 43.60 & 42.45 & 37.53 & 60.56 & 35.30 & 42.96 \\
\hline DUN 22 X Pam 117 & 46.04 & 41.76 & 31.27 & 35.34 & 43.20 & 47.43 & 33.76 \\
\hline DUN 22 X CSR 27 & 45.80 & 71.22 & 66.54 & 51.64 & 53.31 & 47.43 & 59.71 \\
\hline DUN 22 X DUN 6 & 34.72 & 51.63 & 54.49 & 50.12 & 55.41 & 35.30 & 44.61 \\
\hline DUN 22 X S 8 & 44.42 & 69.62 & 66.74 & 46.63 & 46.46 & 50.46 & 55.90 \\
\hline DUN 22 X N 5 & 36.57 & 59.59 & 54.49 & 47.46 & 43.87 & 53.73 & 46.83 \\
\hline DUN 6 X Pam 117 & 52.81 & 59.65 & 54.67 & 47.27 & 36.70 & 26.86 & 44.06 \\
\hline DUN 6 X CSR 27 & 47.45 & 51.63 & 42.98 & 53.25 & 50.30 & 41.35 & 46.15 \\
\hline DUN 6 X DUN 22 & 24.56 & 40.41 & 42.45 & 52.64 & 40.37 & 44.41 & 32.02 \\
\hline DUN 6 XS 8 & 40.90 & 29.19 & 42.45 & 49.22 & 60.04 & 62.62 & 45.55 \\
\hline DUN 6 X N 5 & 57.42 & 50.03 & 54.49 & 49.83 & 38.70 & 53.51 & 51.10 \\
\hline S 8 X DUN 22 & 49.04 & 51.22 & 41.87 & 36.46 & 38.50 & 59.57 & 42.02 \\
\hline S 8XDUN 6 & 57.90 & 38.81 & 54.49 & 55.51 & 38.07 & 62.62 & 52.09 \\
\hline N 5 X DUN 22 & 51.58 & 57.94 & 60.52 & 56.10 & 42.15 & 60.00 & 57.12 \\
\hline N 5 X DUN 6 & 51.82 & 37.18 & 42.45 & 44.63 & 37.63 & 53.51 & 40.40 \\
\hline
\end{tabular}


Crossef DOI: 10.21276/SSR-IIJLS.2020.6.6.4

\begin{tabular}{llllllll}
\hline SH6XNB4D2 & 60.07 & 37.34 & 47.69 & 62.21 & 60.80 & 56.24 & 58.47 \\
CSR6XCSR26 & 32.07 & 47.94 & 48.47 & 55.27 & 53.78 & 50.54 & 45.25 \\
CSR2XCSR27 & 40.22 & 49.76 & 48.83 & 53.63 & 60.31 & 50.51 & 50.60 \\
\hline
\end{tabular}

\section{DISCUSSION}

The phenomenon of heterosis has been commercially exploited in agriculture, horticulture, animal husbandry, piggery, poultry and sericulture. Heterosis is the phenotypic result of gene interaction in heterozygotes and the degree of heterosis increases as the genetic similarities between two parent's decreases. By exploiting the heterosis, various silkworm-breeding efforts have significantly transformed the sericulture scenario by increased qualitative and quantitative production. Silkworm is the only animal, where hybrids are used compulsorily on the commercial scale. Systematic breeding approaches adapted by various silkworm breeders in different sericulture advanced countries ${ }^{[9-13]}$ have contributed to synthesize silkworm (B. mori L) genotypes of desirable constitution and improvement of several quantitative and qualitative traits of economic value.

In the present study, among the 30 hybrids, only 13 hybrids showed a variable amount of relative heterosis. Hybrids having CSR 27, Pam 117, S8 and N5 as one of the parents have shown higher heterosis. The superiority of these parents has also been confirmed in a study on the identification of suitable silkworm foundation crosses ${ }^{[14]}$. Evaluation of the genetic potential of some genotypes of silkworm has also confirmed the superiority of PAM117 when studied under temperate climatic conditions ${ }^{[15] .}$

The hybrids CSR 27 X Pam 117 and its reciprocal Pam 117 $X$ CSR27 have shown the average heterosis values as 4.22 and 3.44 respectively. The top-ranking six hybrids are 58 X CSR27; CSR27 X Pam 117; Pam 117 X N5; Pam 117 X CSR27; S8 X Pam 117 and CSR27 X DUN22. These hybrids have scored $>50$ index value in most of the parameters studied. Among the 30 hybrids, only 8 hybrids showed a variable amount of heterobeltiosis. Hybrids having CSR 27, Pam 117, S8, N5 and DUN22 as one of the parents have shown higher heterobeltiosis. The hybrids CSR $27 \mathrm{X}$ Pam 117 and its reciprocal Pam 117 X CSR27 have shown the average heterobeltiosis values as 1.99 and 1.24 respectively. Similar studies based on heterosis and heterobeltiosis has also been carried out for identification of silkworm foundation crosses ${ }^{[16]}$. The top-ranking six hybrids are S8 X CSR27; Pam 117 X N5; CSR27 X Pam 117; Pam 117 X CSR27; S8 X Pam 117 and CSR27 X DUN22. These hybrids have scored $>50$ index value in most of the parameters studied. The results obtained on heterosis, heterobeltiosis and Evaluation index conform to the observations of earlier workers $^{[17-31]}$.

\section{CONCLUSIONS}

Hybrids having CSR 27, Pam 117, S8 and N5 as one of the parents have shown higher heterosis and heterobeltiosis. Among the hybrids S $8 \times$ CSR 27 and CSR $27 \times$ Pam 117 have shown higher relative heterosis and heterobeltiosis. The results have also been confirmed through evaluation index values calculated on relative heterosis and heterobeltiosis.

These hybrids can be exploited commercially during summer season under Kashmir climatic conditions.

\section{ACKNOWLEDGMENTS}

Authors wish to express a deep sense of gratitude to Central Silk Board, Ministry of Textiles, Govt. of India and Central Sericultural Research and Training Institute, Pampore, Jammu and Kashmir for providing financial assistance and laboratory facilities to carry out the research work.

\section{CONTRIBUTION OF AUTHORS}

Research concept- Mir Nisar Ahmad, Bharath Kumar Neelaboina

Research design- Mir Nisar Ahmad, Shivkumar

Supervision- Materials- Mir Nisar Ahmad

Data collection- Mir Nisar Ahmad, Kiran R

Data analysis and interpretation- Mir Nisar Ahmad, Bharath Kumar Neelaboina

Writing article- Mir Nisar Ahmad

Article review- Bharath Kumar Neelaboina, Shivkumar, Kiran R

Article editing- Sukhen Roy Chowdhury, Bharath Kumar Neelaboina

Final approval- Sukhen Roy Chowdhury

\section{REFERENCES}

[1] Chauhan TPS, Tayal MK. Mulberry Sericulture. In: Industrial Entomology (Eds) Omkar. Springer, Singapore. 2017; 197-63. 
[2] Fan-Sun F, Ye CJ, Li B, Wang T, Fan T. Application of mass spectrometry in silkworm research. Biomed Chromatogr, 2018; 33(4): e4476. doi: 10.1002/bmc.4476.

[3] Nagaraju J, Goldsmith MR. Silkworm genomicsprogress and prospects. Curr Sci., 2002; 83(4): 41525.

[4] Jingade $A H$, Vijayan $K$, Somasundaram $P$, Srinivasababu GK, Kamble CK. A review of the implications of heterozygosity and inbreeding on germplasm biodiversity and its conservation in the silkworm, Bombyx mori. J Insect Sci., 2011; 11: 8.

[5] Sahaf KA, Bhat SA, Mir NA. Sericulture in North-west India with special reference to the temperate regionproblems and prospects. National seminar on sericulture development in the temperate regionproblems and prospects, 2016; pp. 34-38.

[6] Nisar M, Chisti MZ, Khan MA. Studies on the identification of summer-specific silkworm Bombyx mori L. hybrids under temperate climatic conditions of Jammu and Kashmir. India. J Int Acad Res Multidisci., 2013; 1(3): 1-14.

[7] Krishnaswamy S. New Technology of Silkworm Rearing. Central Silk Board Publication, Bangalore Karnataka. 1990; 23.

[8] Mano Y, Nirmal KS, Basavaraja HK, MalReddy N, Datta RK. A new method to select promising silkworm breed/hybrid combinations. Indian Silk, 1993; 31(10): 53.

[9] He Y, Sima YL, Jiang D, Dai P. Breeding of the silkworm varieties for summer and autumn rearing, "Xuhua", "Qiuxing" and their hybrids. Acta Sericologia Sinica., 1991; 17: 200-07.

[10]Mano Y, Ohyanagi M, Nagayasu K, Murakami A. Breeding of sex-limited larval marking silkworm [Bombyx mori] race, N147xC145. Bull Nat Inst Seril Entomol Scie., 1991; 2: 1-29.

[11]Hong KW, Hwang SJ, Ryu KS, Choi SR, Kim KY, et al. Breeding of Bunongjam, a high silk yielding silkworm variety for spring rearing season. Research Reports of the Rural Development Administration, Farm Management, Agricultural Engineering, Sericulture and Farm Products Utilization, 1992; 34: 30-35.

[12]Thiagarajan V, Bhargava SK, Ramesh BM, Nagaraj B. Difference in seasonal performance of 26 strains of silkworm Bombyx mori. (Bombycidae). J Lep Soc., 1993; 47: 321-37.
[13]Datta RK, Basavaraja HK, Mal RN, Nirmal KS, Suresh $\mathrm{KN}$, et al. Breeding of new productive bivoltine hybrid, CSR12 x CSR6 of silkworm, Bombyx mori L. Int J Indust Entomol., 2001; 3: 127-33.

[14]Bharath KN, Shivkumar, Mir NA, Ghosh MK. Evaluation of elite bivoltine silkworm (Bombyx mori L.) foundation crosses suitable for temperate region of Jammu \& Kashmir, India. Int J Curr Microbiol App Sci., 2019; 8(1): 2980-90.

[15]Mir NA, Bharath KN, Shivkumar, Ghosh MK. Evaluation of Genetic Potential of some Genotypes of Silkworm Bombyx mori L under Temperate Climatic conditions. J Crop Weed, 2018; 14(3): 88-93.

[16]Bharath KN, Shiv K, Mir NA, Kiran R, Chowdhury SR. Heterosis Studies on Sustainable Bivoltine Silkworm (Bombyx mori L.) Foundation Crosses Suitable for Kashmir Valley. Res J Agri Sci., 2020; 11(2): 333-40.

[17]Basavaraja HK, Nirmal KS, Suresh KN, MalReddy N, Kshama G,et al. New productive bivoltine hybrids. Indian Silk, 1995; 34: 5-9.

[18]Basavaraja HK, Ashwath SK, Suresh KN, MalReddy N, Kalpana GV. Silkworm Breeding \& Genetics. Central Silk Board Publication, Bangalore, Karnataka, 2005; 523.

[19]Bharath KN, Shiv K, Mir NA, Ghosh MK. Studies on the Performance of Some Silkworm, Bombyx mori L, Breeds in Temperate Region of Jammu and Kashmir, India. Int J Curr Microbiol App Sci., 2018; 7(11): 219201.

[20]Bharath KN, Shiv K, Mir NA, Ghosh MK. Evaluation of bivoltine mulberry silkworm Bombyx mori L breeds suitable for temperate region of Jammu and Kashmir, India. J Entomol Zool Stud., 2019; 7(1): 42327.

[21]Choudhary N, Singh R. Evaluation of few polyvoltine $x$ bivoltine hybrids of the silkworm Bombyx mori $\mathrm{L}$. Indian J Seric., 2006; 45: 62-65.

[22] Lakshmi $H$, Ramesh BM, Saha AK, Chandrashekharaiah M, Bindroo BB. Studies on the season wise evaluation of productive Bivoltine Silkworm (Bombyx mori L.) Hybrids in Tropical Conditions. Int J Int Sci Inn Tech Sec., 2012; 1(1): 20 30.

[23]Mir NA, Shiv K, Bharath KN, Ghosh MK. Evaluation of selected genotypes of bivoltine silkworm Bombyx mori L. during different seasons of Kashmir. J Entomol Zool Stud., 2019; 7(1): 1030-35. 
[24]Naseema BA, Basavaraja HK, Rao PS, Rekha M, Ahsan MM. Identification of bivoltine silkworm hybrids suitable for tropical climate. Indian J Seric., 2000; 39: 24-29.

[25]Nirmal KS, Murthy P, Moorthy SM. Heterosis Studies in Selected Quantitative Traits in Silkworm, Bombyx mori L. Afr J Basic Appl Sci., 2010; 2(5-6): 135-43.

[26]Nisar M, Khan MA, Quadir SM. Evaluation for identification of spring specific silkworm Bombyx mori L. hybrids for commercial exploitation under Kashmir climatic conditions. In: $20^{\text {th }}$ Congress of the Intl. Seric. Commission, Bangalore, India. 2005; 1: 351-56.

[27]Nisar M, Khan MA, Quadir SM. Studies on identification of new silkworm Bombyx mori $\mathrm{L}$. hybrids and their introduction in field for commercial exploitation. Science for Better Tomorrow, University of Kashmir, 2008; 373-76.
[28]Nisar M, Khan MA, Quadir SM, Siddiqui AA. Breeding of Spring Specific Bivoltine Silkworm hybrid SBGP5 $X$ SBGP22. Mulberry Sericulture- Problems and Prospects, APH Publishing Corporation, New Delhi, 2008; 95-104.

[29]Shiv K, Bharath KN, Mir NA, Shakeel A, Ravindra MA, et al. Studies on improvement of quantitative traits of silkworm, Bombyx mori L. during autumn season under temperate climatic conditions of Kashmir. J Entomol Zool Stud., 2018; 6(4): 677-82.

[30]Suresh KN, Basavaraja HK, Kalpana GV, Mal RN, Dandin SB. Effect of high temperature and high humidity on the cocoon shape and size of parents, foundation crosses, single and double hybrids of bivoltine silkworm, Bombyx mori L. Indian J Seric., 2003; 42: 35-40.

[31]Suresh KN, Basavaraja HK, Joge PG, Mal RN, Kalpana $\mathrm{GV}$ et al. Development of new robust bivoltine hybrid (CSR46 x CSR47) of Bombyx mori L for the tropics. Indian J Seric., 2006; 45: 21-29. 